University of Wollongong

Research Online

Faculty of Engineering - Papers (Archive)

Faculty of Engineering and Information

Sciences

$1-4-2005$

\title{
Chain Entanglement in Thin Freestanding Polymer Films
}

L. Si

McMaster University, Canada

M. V. Massa

McMaster University, Canada

K. Dalnoki-Veress

McMaster University, Canada

H. R. Brown

University of Wollongong, hbrown@uow.edu.au

R. A. Jones

University of Sheffield

Follow this and additional works at: https://ro.uow.edu.au/engpapers

Part of the Engineering Commons

https://ro.uow.edu.au/engpapers/277

\section{Recommended Citation}

Si, L.; Massa, M. V.; Dalnoki-Veress, K.; Brown, H. R.; and Jones, R. A.: Chain Entanglement in Thin Freestanding Polymer Films 2005.

https://ro.uow.edu.au/engpapers/277

Research Online is the open access institutional repository for the University of Wollongong. For further information contact the UOW Library: research-pubs@uow.edu.au 


\title{
Chain Entanglement in Thin Freestanding Polymer Films
}

\author{
Lun $\mathrm{Si}^{1}{ }^{1}$ Michael V. Massa, ${ }^{1}$ Kari Dalnoki-Veress, ${ }^{1, *}$ Hugh R. Brown, ${ }^{2}$ and Richard A. L. Jones ${ }^{3}$ \\ ${ }^{1}$ Department of Physics \& Astronomy and the Brockhouse Institute for Materials Research, McMaster University, \\ Hamilton, ON, Canada \\ ${ }^{2}$ Steel Institute, University of Wollongong, Wollongong, Australia \\ ${ }^{3}$ Department of Physics \& Astronomy, University of Sheffield, Sheffield, United Kingdom
} (Received 16 July 2004; published 1 April 2005)

\begin{abstract}
When a thin glassy film is strained uniaxially, a shear deformation zone (SDZ) can be observed. The ratio of the thickness of the SDZ to that of the undeformed film is related to the maximum extension ratio, $\lambda$, which depends on the entanglement molecular weight, $M_{e}$. We have measured $\lambda$ as a function of film thickness in strained freestanding films of polystyrene as a probe of $M_{e}$ in confinement. It is found that thin films stretch further than thick films before failure, consistent with the interpretation that polymers in thin films are less entangled than bulk polymers, thus the effective value of $M_{e}$ in thin films is significantly larger than that of the bulk. Our results are well described by a conceptually simple model based on the probability of finding intermolecular entanglements near an interface.
\end{abstract}

DOI: 10.1103/PhysRevLett.94.127801

How polymers are influenced in confined systems has been a main focus of polymer science-especially now that systems with very small dimensions are becoming easier to manufacture. In the bulk melt state, polymers are considered ideal Gaussian chains which represent random walks with a length scale dependent on the molecular weight, $M$, of the polymer [1]. A natural length scale for ideal chains is the root-mean-squared end-to-end distance, $R_{e e} \sim M^{1 / 2}$. A qualitative picture of the polymer melt is that a polymer chain explores a pervaded volume, $V_{p} \sim$ $R_{e e}^{3}$, due to random thermal motions. Within this same volume there are many other polymers and many interactions between a specific polymer chain and other chains. A fraction of these interactions restrict the mobility of a polymer chain resulting in entanglements, which can be thought of as knots or crossings in the system. Entanglements are at the root of many remarkable polymeric properties like very high melt viscosities, transient rubberlike behavior, and toughness [1].

The nature of entanglements in confined systems is not yet understood and we must be careful to distinguish between the idea of chain overlap and chain entanglement. This is most easily understood from a discussion of thin films which originates in the text of de Gennes (p. 61 of Ref. [1]). A nonadsorbing interface can be approximated as a reflective boundary [2], which implies that the ideal chains are only perturbed in the direction normal to the interface (i.e., the random walk is unperturbed in the plane of the film). The weak perturbation of chain dimensions in the lateral direction has been validated by Müller [3] and Vacatello [4] with Monte Carlo simulations and experimentally by Jones and co-workers [5]. As the thickness of the film, $h$, is decreased below the length scale of the molecule, $h \ll R_{e e}$, the volume pervaded by a single molecule decreases, while the overall monomer density remains constant. With increasing confinement, segments belonging to other chains are increasingly excluded from
PACS numbers: 61.41.+e, 62.20.Mk, 68.60.-p, 82.35.Lr

the pervaded volume, resulting in a reduced interchain entanglement density, while the total entanglement density remains constant.

Though the exact conformations that represent entanglements are not known, the statistical nature of entanglements in bulk systems is very well explained by chain packing models [6-8]. In such models the entanglement molecular weight, $M_{e}$, is defined through the ratio of the pervaded volume of a chain, $V_{p}$, to the volume occupied by that chain, $V_{c}$. The volumes can be expressed as $V_{p}=$ $A R_{e e}^{3}=A(M / m)^{3 / 2} a^{3}$ and $V_{c}=(M / m) a^{3}$, where $A$ is a constant of order $1, m$ is the mass of a monomer, and $a$ is a length associated with the monomer. The argument made in the chain packing models is that an entanglement occurs when $M$ is such that $\sim 2$ chains exist in the same pervaded volume (the precise value of this number varies depending on the details of the model). As chains get larger, they interact with more other chains, thus $M_{e}$ corresponds to some ratio of $V_{p} / V_{c} \sim 2$. Though it is not explicitly stated in these models, we note that an entanglement is then defined through interactions with other chains-i.e., through interchain entanglements. In the bulk this represents a subtle and minor detail, but in confinement the situation is different.

Consider a polymer chain of mass $2 M_{e}$. This chain could be confined to the pervaded volume that would be occupied in the bulk by a chain of mass $M_{e}$. Thus a chain that was well-entangled in the bulk would, in this confined situation, be only marginally entangled with other chains. Yet the total number of entanglements as defined by packing models remains constant; what in the bulk are interchain entanglements are converted in confinement to selfentanglements. The conclusion to be drawn from this argument is that to a first approximation, the total entanglement density, $\nu \sim 1 / M_{e}$, which is made up of the sum of the self-entanglements and interchain entanglements, remains constant. It is the fraction of self-entanglements that in- 
creases in a confined system because the pervaded volume is smaller in confinement than in the bulk. The ideas presented here are an extension of the packing models to confined geometries, and in spirit similar to models presented to describe entanglements near an interface [9] and the overlap between chains in a thin film [1].

When strain is applied to a polymer glass, failure can occur though crazing (small nanoscopic voids which form in polymeric glasses under strain) [10-16]. In thin films a shear deformation zone (SDZ) can be observed rather than a craze. The SDZ is a thinning or neck that forms in the film. The SDZ appears qualitatively different from a craze observed in bulk systems because the constraint in the normal direction of a film is relaxed [10,11,13-15]. The extension ratio, $\lambda$, is an important property of a craze or SDZ and is the ratio of the width of the material that went into forming the deformation to the width of the deformed region after an applied strain. Many of the details regarding crazing and shear deformation are well understood due to the pioneering work by Kramer and co-workers. Donald and Kramer found that by mixing two miscible polymers with different entanglement molecular weights, it was possible to vary $M_{e}$ systematically, resulting in corresponding changes in $\lambda$ [12]. These experiments proved conclusively the link between $M_{e}$ and $\lambda$. Donald and Kramer found the results to agree quantitatively with what was expected from a model based on the distance between two entanglement points prior to and after deformation (see [12] and the reviews [13,14]). Rottler and Robbins also established this link between $M_{e}$ and $\lambda$ using simulations [16].

In this Letter we present a study which makes use of the fact that the extension ratio can be used as a relatively large scale observable to probe the molecular geometry of entanglements, $\lambda^{2} \sim M_{e}[12-14,16]$. Measurements of $\lambda$ were performed in thin freestanding films (membranes) of polystyrene (PS). We found that by measuring $\lambda$ it was possible to infer that the effective entanglement molecular weight $M_{\text {eff }}$ increases as the film thickness, $h$, is decreased. Furthermore, a model based on chain packing [6-9] is presented which is in remarkable agreement with the experiments.

Thin PS films with thicknesses ranging from $30 \mathrm{~nm}$ to $650 \mathrm{~nm}$ were spincast out of toluene onto freshly cleaved mica. Three molecular weights of PS (Polymer Source Inc., Canada) were used and measured to be 623, 828, and $1062 \mathrm{~kg} / \mathrm{mol}$ (the polydispersity index was less than 1.1 for all three). The Mica-PS samples were annealed at $115^{\circ} \mathrm{C}$ at a pressure of $\sim 10^{-6}$ Torr for $12 \mathrm{~h}$ to remove residual stress and solvent in the film. The samples were cooled to room temperature at a rate of $\sim 1{ }^{\circ} \mathrm{C} / \mathrm{min}$. The film, cut into small pieces, was floated onto a clean water (Milli-Q) surface and a section picked up with a two part stainless steel sample holder with a $1.5 \mathrm{~mm}$ gap. The sample was then dried in a clean air environment and attached over an inverted microscope with one side fixed and the other attached to a computer controlled translation stage (Newport MFN25CC, ESP300). For all the results presented, the translation stage velocity was $0.3 \mu \mathrm{m} / \mathrm{s}$ resulting in a strain rate of $2 \times 10^{-4} \mathrm{~s}^{-1}$ and the final gap was $1.8 \mathrm{~mm}$. After the strain was applied, the sample and sample holder were removed from the microscope and placed onto a cleaned Si wafer while still under tension. The surface forces pull the sample onto the Si substrate and stabilize the film [see Fig. 1(a)]. The width of the neck (of order $\sim 10 \mu \mathrm{m}$ ) is much greater than the thickness of the neck (of order $\sim 10 \mathrm{~nm}$ ), such that a transfer to Si results in the neck being in contact with the Si. This fact could be verified by atomic force microscopy (AFM) as well as optical microscopy.

Figure 1(b) is representative of the measurements that were performed. A scratch was made in the film such that an AFM scan could simultaneously be used to measure both the height of the film $h$ and the depth of the neck $d$. For each film a measure of the ratio of the neck thickness to the film thickness was obtained by averaging several scan lines on at least 5 AFM images at different locations along the neck. Figure 2 is a plot of the ratio $h_{c} / h$ as a function of $h$ for all three molecular weights. It is clear that a constant value of $h_{c} / h$ is obtained for thick films which is equivalent for all values of $M$. However, as the film thickness is decreased below $\sim 100 \mathrm{~nm}$, there is a significant decrease in $h_{c} / h$. Furthermore, the decrease in $h_{c} / h$ is monotonic and the effect is shifted to thinner films for smaller polymers - a clear indication that chain confinement effects are important.

Figure 3 shows a diagram of a polymer chain in a neck prior to and after a strain. The theoretical maximum value of $\lambda$ is the ratio of the distance between two entanglement points for ideal Gaussian chains before and after a strain, $\lambda \sim l_{e} / d \sim M_{e} / \sqrt{M} \sim \sqrt{M_{e}} e$. The validity of this model has been well tested experimentally by Donald and Kramer [12-14] and using simulations by Rottler and Robbins [16]. If we make the assumption that the density of material in the neck and in the film is equivalent (conserved vol- a)
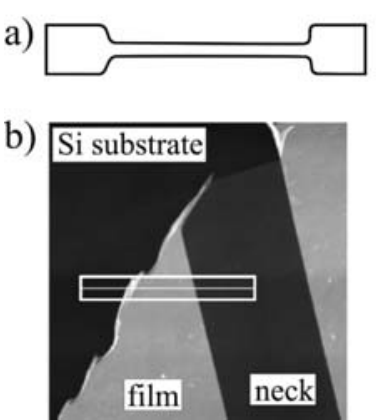
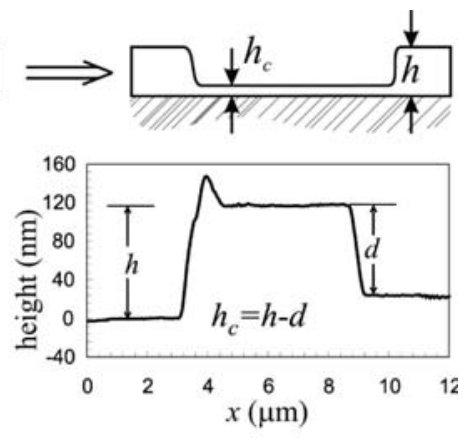

FIG. 1. (a) A freestanding membrane that has been strained is transferred to a Si substrate. (b) AFM is used to scan the sample in order to measure the thickness of the film and the deformed neck. The image is $25 \mu \mathrm{m} \times 25 \mu \mathrm{m}$ wide; $z$ range is $140 \mathrm{~nm}$. The scan lines are averaged in a region (white bar) that simultaneously shows the substrate, film, and neck. 


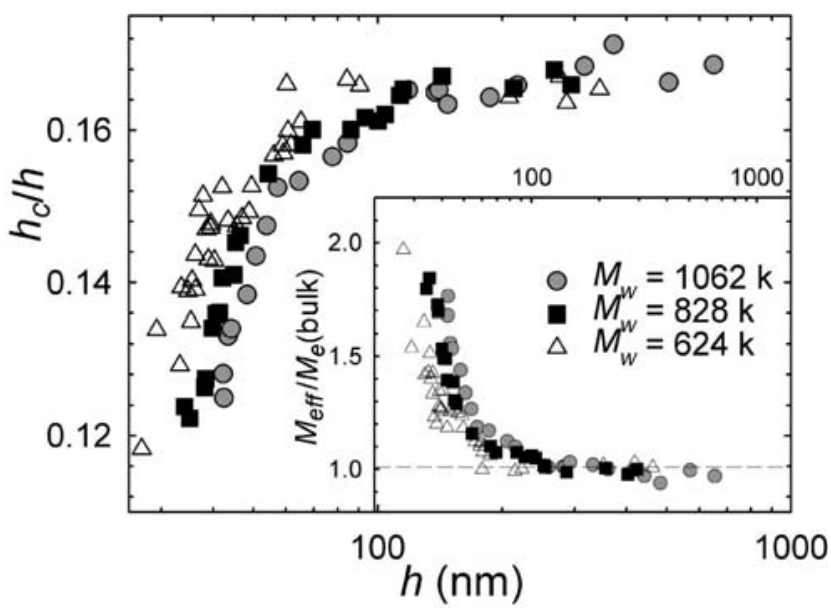

FIG. 2. Plot of the ratio of the neck thickness to the film thickness, $h_{c} / h$, as a function of $h$ for three molecular weights. In the inset the data are replotted as the normalized value of the effective entanglement molecular weight, $M_{\mathrm{eff}} / M_{e}$ (bulk).

ume), then $h_{c} / h=1 / \lambda \sim 1 / \sqrt{M_{e}}$ [17]. Applying this relationship to the case of thin films, it is possible to obtain the effective entanglement molecular weight, $M_{\mathrm{eff}}$. In the inset of Fig. 2 we plot $M_{\text {eff }}$, normalized to the bulk as a function of $h$. The effective entanglement molecular weight in the thinnest films is greater by a factor of $\sim 2$. Simply stated, a thin film will stretch further than a thick film before failure because the network is more loosely entangled.

The results presented thus far can be understood from a simple model, which represents a first approximation to a complex problem. The model first of all requires the assumption that self-entanglements and interchain entanglements do not contribute equally to the integrity of the network for these strain experiments. Certainly in the limit of very thin films, where there is no chain overlap and hence only self-entanglements, the network must be very fragile. As discussed above, we also assume that the total entanglement density remains constant and that it is the fraction of self-entanglements that increases near an interface. We can then write the effective entanglement density as $\nu_{\text {eff }}=\nu P_{\text {inter }}=\nu\left(1-P_{\text {self }}\right)$, where $P_{\text {inter }}$ is the probability of finding interchain entanglements and $P_{\text {self }}$ is the probability of self-entanglements. In Fig. 4(a) we show a schematic of a thin film. A chain near the interface is perturbed as it is reflected by the boundary [2]. If a chain's pervaded volume in confinement were half that of an unperturbed chain, then the probability of a self-

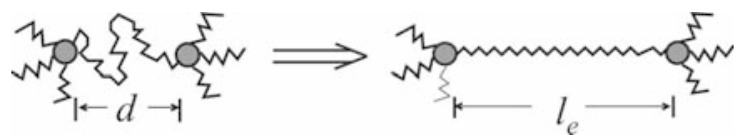

FIG. 3. Diagram of the Gaussian chain between two entanglement points before and after an applied strain. entanglement would be $2 P$, where $P$ represents the probability of a self-entanglement occurring in unperturbed chains. Thus

$$
\nu_{\mathrm{eff}}=\nu\left(1-P \frac{V_{b}}{V_{p}}\right)
$$

where $V_{b}$ is the average volume pervaded by a "bulk" chain (unperturbed) and $V_{p}$ is the volume pervaded by a chain in general (i.e., in the bulk $V_{p}=V_{b}$, but at an interface $V_{p}$ is reduced to the portion of the sphere that is within the film). For unperturbed bulk chains we define $V_{b}=$ $4 \pi R^{3} / 3$, where the radius of the unperturbed chain volume is $R=c R_{e e}$, and $c$ is a constant. It is expected that since $R_{e e}$ is a natural length scale for the size of the polymer that $c \sim 1$.

In order to calculate the effective entanglement density in the film we assume that we can average Eq. (1) over the film thickness $h$. This is an approximation; in reality we expect that $\nu_{\text {eff }}$ would be less near an interface and tend towards the bulk value away from an interface (a similar idea is explored in [9]). From Eq. (1)

$$
\frac{\left\langle\nu_{\mathrm{eff}}\right\rangle}{\nu}=1-P\left\langle\frac{V_{b}}{V_{p}}\right\rangle=1-\frac{P}{h} \int_{0}^{h} \frac{V_{b}}{V_{p}(x)} d x .
$$

We define the dimensionless film thickness $H=h / R$ and solve Eq. (2) for three cases: $h<R, R<h<2 R$,

a)

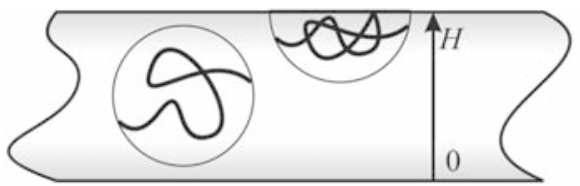

b)

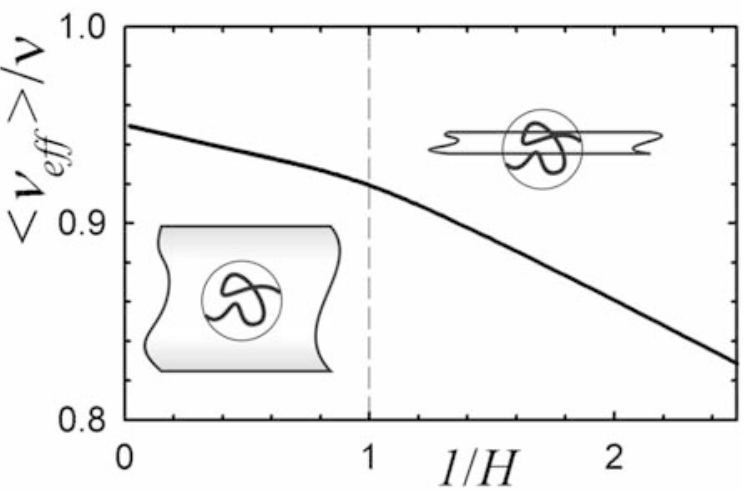

FIG. 4. (a) Diagram of a polymer chain in a film. The pervaded volume of a chain is reduced near an interface. (b) Plot of the normalized film-averaged effective entanglement density vs the inverse film thickness (the plot is for $P=0.05$; similar results are obtained for other values of $P$ ). 


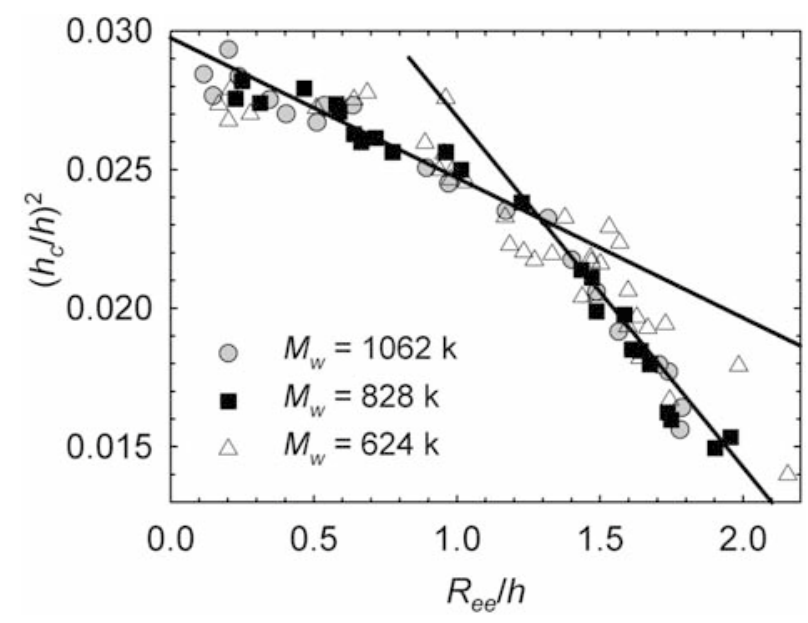

FIG. 5. Plot of the experimentally obtained value of $\left(h_{c} / h\right)^{2}$ as a function of the dimensionless ratio $R_{e e} / h$ for three molecular weights. According to the model $\left(h_{c} / h\right)^{2} \sim\left\langle\nu_{\text {eff }}\right\rangle$ [see Fig. 4(b)].

and $h>2 R$ :

$$
\frac{\left\langle\nu_{\mathrm{eff}}\right\rangle}{\nu}=\left\{\begin{array}{cl}
1+\frac{16 P}{H^{2}}\left[\frac{\operatorname{atanh}\left(\frac{3 H}{\sqrt{36-3 H^{2}}}\right)}{\sqrt{36-3 H^{2}}}\right] & H<1 . \\
1-\frac{8 P}{3 H^{2}}\left[\frac{6 \tanh \left(\frac{3 H-6}{\sqrt{36-3 H^{2}}}\right)}{\sqrt{36-3 H^{2}}}+\right. & \\
\left.\frac{H}{3} \ln \left(\frac{2 H}{3-H}\right)+H-1\right] & 1<H<2 \\
1-P\left(1+\frac{0.5656}{H}\right) & H>2 .
\end{array}\right.
$$

In Fig. 4(b) the calculated value of $\left\langle\nu_{\text {eff }}\right\rangle$ is plotted as a function of $1 / H$. Clearly there are two regimes that are nearly linear in $1 / H$. For thick films only the interfacial region is affected, resulting in a linear dependence of $\left\langle\nu_{\text {eff }}\right\rangle$ on $1 / H$. As the films get thinner, polymer chains interact with both interfaces and the effect on $\left\langle\nu_{\text {eff }}\right\rangle$ becomes more severe. A direct comparison to the data is possible through the crossover point at $H \sim 1$, as well as the contrast in the slopes of the two regimes $\sim 2.2$ [18].

Recalling that $\nu_{\text {eff }} \sim\left(h_{c} / h\right)^{2}$, we show the data plotted as $\left(h_{c} / h\right)^{2}$ versus $R_{e e} / h$ in Fig. 5 (the scaling predicted by the model). The agreement between the model and the data is excellent: (1) the data clearly break up into two nearly linear regimes for all values of $M$ studied; (2) all three molecular weights collapse as suggested by the model $\left(1 / H=R / h=c R_{e e} / h\right)$; (3) the contrast in the slope of the two linear regimes is $\sim 2.7$ while the model value is $\sim 2.2$; and (4) from the crossover of the two regimes, it is found that the pervaded volume of a polymer chain is $R \sim$ $R_{e e} / 1.3$ (note that the size of the pervaded volume is what one might reasonably expect, $R=c R_{e e}, c \sim 1$ ).

The results presented are the first measurements that probe polymer entanglement in confined systems. We can conclude with confidence that thin films respond to a strain that is consistent with a network with an entanglement density that is significantly reduced. The model, which is in agreement with the data, is a simple geometrical extension of ideas based on chain packing [6-8], coupled with predictions that there is reduced chain overlap near interfaces [1,9]. The model is a first approximation to a very complex problem which may provide a starting point for more rigorous treatments. If we accept the model, then the following conclusions can be drawn: (1) we must distinguish between self-entanglements and interchain entanglements; (2) the total entanglement density is constant, but the proportion of self-entanglements increases near interfaces; and (3) the results provide a measure of the pervaded volume of a polymer chain.

We thank Athene Donald, James Forrest, Stephan Herminghaus, Ed Kramer, Greg McKenna, Günter Reiter, and Jens-Uwe Sommer for valuable discussions. We are grateful to Greg Bahun for the molecular weight determination. Financial support from NSERC, PREA, CFI, and OIT of Canada is gratefully acknowledged.

*Electronic address: dalnoki@mcmaster.ca

[1] P.-G. de Gennes, Scaling Concepts in Polymer Physics (Cornell University Press, Ithaca, New York, 1979).

[2] A. J. Silberberg, J. Colloid Interface Sci. 90, 86 (1982).

[3] M. Müller, J. Chem. Phys. 116, 9930 (2002).

[4] M. Vacatello, Macromol. Theory Simul. 13, 30 (2004).

[5] R. L. Jones et al., Nature (London) 400, 146 (1999).

[6] Y.-H. Lin, Macromolecules 20, 3080 (1987).

[7] T. A. Kavassalis and J. Noolandi, Macromolecules 21, 2869 (1988).

[8] L. J. Fetters et al., Macromolecules 27, 4639 (1994).

[9] H. R. Brown and T.P. Russell, Macromolecules 29, 798 (1996).

[10] A. M. Donald et al., J. Mater. Sci. 16, 669 (1981).

[11] T. Chan et al., J. Mater. Sci. 16, 676 (1981).

[12] A. M. Donald and E. J. Kramer, Polymer 23, 461 (1982).

[13] E. J. Kramer, Adv. Polym. Sci. 52/53, 1 (1983).

[14] E. J. Kramer and L. L. Berger, Adv. Polym. Sci. 91/92, 1 (1990).

[15] T. N. Krupenkin and G.H. Fredrickson, Macromolecules 32, 5029 (1999).

[16] J. Rottler and M. O. Robbins, Phys. Rev. E 68, 011801 (2003).

[17] This is a good approximation since an upper limit of the density of the neck is the density of crystalline PS. Syndiotactic PS has a density that is only a few \% higher than amorphous PS.

[18] Since the thin film regime is not strictly linear we need to evaluate the slope contrast and crossover by finding the tangent to the curve. Because the curve is nearly linear, the choice of tangent affects the results only slightly $(1 / H=$ 1.5 was used). 